\title{
Fat intake leads to differential response of rat adipocytes to glucose, insulin and ascorbic acid
}

Short title: Fat intake leads differential adipocyte response

Diego F. Garcia-Diaz ${ }^{1}$, Javier Campion ${ }^{1}$, Arianna V. Arellano ${ }^{1}$, Fermin I. Milagro ${ }^{1}$, Maria J. Moreno-Aliaga ${ }^{1}$ and J. Alfredo Martinez ${ }^{1}$

${ }^{1}$ Department of Nutrition and Food Sciences, Physiology and Toxicology University of Navarra, 31008, Pamplona, Spain

Corresponding author: Prof. J. Alfredo Martinez

Department of Nutrition and Food Sciences,

Physiology and Toxicology

University of Navarra

c/Irunlarrea 1, 31008

Pamplona, Spain

Phone: +34 948425600

Fax: +34948425649

e-mail address: jalfmtz@unav.es 


\section{ABSTRACT}

Antioxidant-based treatments have emerged as novel and interesting approaches to counteract fat accumulation in obesity and associated metabolic disturbances.

Adipocytes from rats that were fed on chow (C) or high fat diet (HFD) for 50 days, were isolated (primary adipocytes) and incubated (72h) on low (LG; $5.6 \mathrm{mM}$ ) or high (HG; $25 \mathrm{mM}$ ) glucose levels, in the presence or absence of $1.6 \mathrm{nM}$ insulin and $200 \mu \mathrm{M}$ vitamin C (VC). Adipocytes from HFD fed animals presented lower insulin-induced glucose uptake, lower lactate and glycerol release, and lower insulin-induced secretion of some adipokines as compared to controls. HG treatment restored the blunted response to insulin regarding apelin secretion in adipocytes from HFD fed rats. VC treatment inhibited the levels of nearly all variables, irrespective of the adipocytes' dietary origin. The HG treatment reduced adipocyte viability, and VC protected from this toxic effect, although more drastically in control adipocytes. Summing up, in vivo chow or HFD intake determines a differential response to insulin and glucose treatments that appears to be dependent on the insulin-resistance status of the adipocytes, while VC modifies some responses from adipocytes independently of the previous dietary intake of the animals.

Keywords: vitamin C, diet-induced obesity, adipose tissue, cell culture, adipokines 


\section{INTRODUCTION}

Excessive body fat accumulation can lead to a number of associated clinical complications such as type 2 diabetes, metabolic syndrome features, cardiovascular diseases and inflammatory disturbances, among others ${ }^{1}$. These adverse clinical manifestations have been linked to impaired production and secretion of endogenous products by enlarged adipocytes or the accompanying macrophages from the white adipose tissue (WAT) stroma-vascular fraction ${ }^{1}$. Actually, several adipocytokines and inflammatory products derived from this tissue (TNF- $\alpha$, IL-6, MCP-1, iNOS) have shown positive correlations with body adiposity ${ }^{2}$. Besides the pro-inflammatory secretion by WAT, this tissue also produces numerous adipokines that may have important local and systemic effects ${ }^{3}$. Among them, leptin, which has been associated with the control of food intake and energy expenditure ${ }^{4}$, and apelin, which is upregulated in obesity and modulated by insulin and TNF- $\alpha$ treatments ${ }^{5,6}$, are considered metabolically relevant.

On the other hand, mitochondrial dysfunctions and over-production of reactive oxygen species (ROS) have been related with obesity ${ }^{7}$. In this sense, elevated oxidative stress in obese patients $^{8}$ and in overweight animal models ${ }^{9}$ has been reported. Thus, induced monocyte migration to the adipose tissue and high secretion levels of some WATsecreted adipokines could be implicated in obesity-associated ROS overproduction ${ }^{9}$, linking the pathogenic secretion pattern of WAT with an enhanced oxidative stress status $^{10}$.

Furthermore, it has been observed that there is a negative association between higher intake of the dietary antioxidant vitamin $\mathrm{C}(\mathrm{VC})$ and the occurrence of hypertension, gallbladder disease, stroke, cancers and atherosclerosis ${ }^{11}$, and also with obesity in 
humans and animals ${ }^{12-14}$. Among the possible beneficial effects of ascorbic acid on obesity-related mechanisms, it has been suggested that this vitamin may: a) modulate adipocyte lipolysis ${ }^{15-17}$, b) regulate the glucocorticoid release from adrenal glands ${ }^{18}, \mathrm{c}$ ) inhibit glucose metabolism and leptin secretion on isolated adipocytes ${ }^{19}$, d) lead to an improvement in hyperglycemia and decrease glycosylation in obese-diabetic mice ${ }^{20}$, and e) reduce the inflammatory response $\mathrm{e}^{21,22}$.

Taking all these findings into account, the present work aimed to analyze whether VC is directly implicated in the metabolic response and in the expression/secretion pattern of leptin and apelin, two important adipokines, in isolated adipocytes from lean or dietinduced obese rats, focusing on the possible differential responses of primary adipocytes that were isolated from high fat fed animals as compared to control lean animals. 


\section{MATERIALS AND METHODS}

\section{Materials}

Dulbecco's modified eagle's medium (DMEM), 100X minimal essential medium (MEM) non-essential amino acids, penicillin/streptomycin, heat-inactivated fetal bovine serum (FBS), 10X MEM, nystatin, Trizol Reagent, and M-MLV reverse transcriptase were obtained from Invitrogen (Paisley, UK). Bovine serum albumin (BSA), HEPES, insulin and 2',7'-dichlorofluorescein (DCFH) were all obtained from Sigma-Aldrich Company (St. Louis, USA). Collagen (Purecol) was purchased from Nutacon (Leimuiden, The Netherlands). Type I collagenase was supplied by Worthington Biochemical Corporation (Lakewood, USA), and vitamin C by Panreac Quimica (Barcelona, Spain). Glycerol-3-phosphate dehydrogenase (GPDH), glycerol kinase (GK), adenosine triphosphate (ATP), and nicotinamide adenine dinucleotide (NAD) were obtained from Roche Diagnostics (Mannheim, Germany).

\section{Animals and dietary treatment}

Eight-week-old male Wistar rats ( $\mathrm{n}=20$; about $200 \mathrm{~g}$ body weight) supplied by the Center for Applied Pharmacobiology Research (CIFA, Pamplona, Spain), were housed in a temperature-controlled room at $21-23{ }^{\circ} \mathrm{C}$ with a 12 hour light cycle (lights goes off at $8 \mathrm{pm}$ ). The animals were assigned into two different dietary groups: a control group $(n=10)$ that was fed on a standard chow diet $(2014$ Tekland Global $14 \%$ Protein Rodent Maintenance Diet; Harlan Iberica, Barcelona, Spain), and a high fat diet fed group (HFD, $n=10)$ that was fed on a high fat diet (D12330 diet, $58 \mathrm{kcal} \%$ fat w/cornstarch Surwit Diet; Research Diets Inc., New Brunswick, USA) as previously described ${ }^{23}$. Experimental diets detailed composition is described in Table 1. Both groups of animals 
had ad libitum access to water and food during the trial, and their body weights and food intakes were recorded daily. After 50 days of dietary treatment the animals were euthanized. The final body weights were recorded, while blood and tissue samples of several WAT depots were collected and weighed. About 3-4 g of epididymal WAT was immediately isolated from total tissue for adipocyte isolation and primary culture experiments. All the procedures were performed according to European, national and institutional guidelines of the Animal Care and Use Committee at the University of Navarra.

\section{Serum measurements}

Circulating glucose (HK-CP kit; ABX diagnostic, Montpellier, France), total cholesterol (Cholesterol CP kit; ABX diagnostic, Montpellier, France), HDL cholesterol (HDL Direct $\mathrm{CP}$ kit; $\mathrm{ABX}$ diagnostic, Montpellier, France), and triglyceride concentrations (Triglycerides CP kit; ABX diagnostic, Montpellier, France) were measured using automated COBAS MIRA equipment (Roche, Basel, Switzerland). Serum leptin (Linco Research, St. Charles, USA), insulin (Mercodia AB, Uppsala, Sweden) and apelin levels (Cusabio Biotech Co., Wuhan, China) were determined by different ELISA kits, with an automated Triturus equipment (Grifols International, Barcelona, Spain) as described by the manufacturers.

\section{Adipocyte isolation}

Primary cultures were obtained according to protocols described elsewhere ${ }^{19}$. Epididymal WAT (3-4 g) of each rat was minced with scissors in HEPES-phosphate buffer (pH 7.4; containing 5 mM D-glucose, $2 \%$ w/v BSA, $135 \mathrm{mM} \mathrm{NaCl}, 2.2 \mathrm{mM}$ $\mathrm{CaCl}_{2} \cdot 2 \mathrm{H}_{2} \mathrm{O}, 1.25 \mathrm{mM} \mathrm{MgSO} \cdot 7 \mathrm{H}_{2} \mathrm{O}, 0.45 \mathrm{mM} \mathrm{KH}_{2} \mathrm{PO}_{4}, 2.17 \mathrm{mM} \mathrm{Na}_{2} \mathrm{HPO}_{4}$, and 10 
mM HEPES). WAT fragments were digested in the same buffer with type I collagenase $\left(1.25 \mathrm{mg} / \mathrm{ml}\right.$ per $0.5 \mathrm{~g}$ tissue) at $37^{\circ} \mathrm{C}$ with gentle shaking for $30 \mathrm{~min}$. The resulting cell suspension was diluted in the buffer, and then the adipocytes were isolated from the undigested tissue by filtration through a $400 \mu \mathrm{m}$ nylon mesh and washed three times with alternated centrifugations at $500 \mathrm{rpm}$ for 6 minutes. Isolated adipocytes were then resuspended in DMEM supplemented with $1 \% \mathrm{v} / \mathrm{v}$ FBS, $100 \mathrm{U} / \mathrm{ml}$ penicillin / 100 $\mu \mathrm{g} / \mathrm{ml}$ streptomycin, $100 \mathrm{U} / \mathrm{ml}$ nystatin and $1 \mathrm{X}$ MEM non-essential amino acids, followed by incubation for $40 \mathrm{~min}$ at $37^{\circ} \mathrm{C}$ in $5 \% \mathrm{CO}_{2}$. The resulting isolated adipocytes (150 $\mu 1$ of a 2:1 mix of packed cells/culture medium) were either used for further measurements or plated on $500 \mu \mathrm{l}$ of a collagen matrix ( $\mathrm{pH} 7 ; 7$ parts collagen : 1 part 10X MEM) in six well culture plates and cultured at $37{ }^{\circ} \mathrm{C} / 5 \% \mathrm{CO}_{2}$.

\section{Isolated adipocyte measurements}

Before primary culture, some measures on the isolated adipocytes were performed. In the first instance, intracellular ROS concentrations were determined using $2^{\prime}, 7^{\prime}$ dichlorofluorescein (DCFH) according to a protocol described elsewhere ${ }^{19}$. Briefly, cells were incubated with $10 \mu \mathrm{M}$ DCFH for 40 minutes in $37^{\circ} \mathrm{C} / 5 \% \mathrm{CO}_{2}$, frozen for at least $1 \mathrm{~h}$ at $-80^{\circ} \mathrm{C}$ and then lysed with $500 \mu \mathrm{l}$ of lysis buffer $(150 \mathrm{mM} \mathrm{NaCl}, 0.1 \%$ v/v Triton and $10 \mathrm{mM}$ Tris). Finally, $200 \mu \mathrm{l}$ of each lysate was plated on a $96-$ well black plate (Labsystems, Barcelona, Spain), and fluorescence intensity was measured with a POLARstar spectrofluorometer plate reader (BMG Labtechnologies, Offenburg, Germany), at an excitation of $485 \mathrm{~nm}$ and an emission of $530 \mathrm{~nm}$. Also, the morphological characteristics of the isolated adipocytes were analyzed. Cells were placed on a Neubauer chamber and coupled on an Olympus CK30 microscope (Olympus Corporation, Barcelona, Spain). Then, pictures were taken with an Olympus 
C-5060WZ Digital Camera (Olympus Corporation, Barcelona, Spain) using a Camedia Adapter C5060-ADUS for IUS Microscope (Olympus Corporation, Barcelona, Spain) at 40X magnification. Pictures in TIFF format with 400x300 pixels resolution were processed with the Scion Image for Windows software (Scion Corporation, Maryland, USA) for cell number and diameter determinations. Finally, mRNA expressions of apelin and leptin in these isolated adipocytes were also measured according to the protocol described below (gene expression assays section).

\section{Adipocytes culture}

After the final 40-50 min incubation, the adipocytes were treated with high (HG, 25 $\mathrm{mM} ; \mathrm{n}=5)$ and low $(\mathrm{LG}, 5.6 \mathrm{mM} ; \mathrm{n}=5)$ glucose concentrations, in the presence or absence of $1.6 \mathrm{nM}$ insulin and $200 \mu \mathrm{M}$ vitamin C (VC). Cells were maintained in an incubator at $37^{\circ} \mathrm{C}$ in $5 \% \mathrm{CO}_{2}$. Aliquots of the culture medium and culture plates were collected at $72 \mathrm{~h}$ and frozen at $-80{ }^{\circ} \mathrm{C}$ for further assays.

\section{Cell viability assay}

The lactate dehydrogenase (LDH) activity $(\mu \mathrm{U} / \mathrm{ml})$ in culture medium is commonly used as an indicator of cell membrane integrity, as well as a measurement of cellular necrosis/apoptosis ${ }^{24}$. Cell viability was measured with the LDH Cytotoxicity Assay Kit according to the supplier's instructions (Cayman Chemical Company, Ann Arbor, USA), and was assessed at the end of the experimental period (72 $\mathrm{h}$ ) in all treatments. 


\section{Culture medium determinations}

Glucose utilization was assessed by measuring the concentration of glucose in the medium in each well at $72 \mathrm{~h}$, and then subtracting it from the initial concentration. Lactate production was calculated as the increase of lactate in the medium at $72 \mathrm{~h}$. Glucose and lactate concentrations were measured with the HK-CP kit from ABX diagnostic (Montpellier, France) and with the L-lactate kit from Randox Laboratories (Crumlin, UK), respectively, using an automated COBAS MIRA equipment (Roche, Basel, Switzerland). Glycerol concentration increase in the medium at $72 \mathrm{~h}$, as a measure of the lipolytic activity, was determined by a colorimetric method as previously published $^{25}$. Briefly, culture media of each sample were incubated with $25 \mu \mathrm{g} / \mathrm{ml}$ GPDH and $250 \mathrm{mU} / \mathrm{ml} \mathrm{GK}$, in the presence of $43.6 \mathrm{mM} \mathrm{MgCl}_{2}, 200 \mathrm{mM}$ glycine, $5.2 \% \mathrm{v} / \mathrm{v}$ hydrazine, $1.24 \mathrm{mM}$ ATP and $573 \mu \mathrm{M}$ NAD for 40 minutes. Finally, absorbance of the samples was measured at $340 \mathrm{~nm}$ in a microplate reader (Multiskan Spectrum, Thermo Fisher Scientific, Waltham, USA). Finally, leptin (sensitivity $\geq 0.04 \mathrm{ng} / \mathrm{mL}$; intra-assay precision $\pm 2.17 \% \mathrm{CV}$; inter-assay precision $\pm 3.40 \% \mathrm{CV}$ ) and apelin (sensitivity $\geq 3.9$ $\mathrm{pg} / \mathrm{ml}$; intra-assay precision $<8 \% \mathrm{CV}$; inter-assay precision $<10 \% \mathrm{CV}$ ) secretions at $72 \mathrm{~h}$ were determined by the ELISA kits as previously indicated in the serum measurements section. All culture medium determinations were corrected for cell viability $^{22}$.

\section{Gene expression assays}

Total RNA was isolated from isolated epididymal adipocytes using Trizol (Invitrogen, Paisley, UK) according to the manufacturer's protocol. Purified total RNA from adipocytes was treated with DNAse (DNAfree kit; Ambion Inc., Austin, USA) and used to generate cDNA with M-MLV reverse transcriptase (Invitrogen, Paisley, UK). 
Relative real-time PCR was performed on an ABI PRISM 7000 HT Sequence Detection System (Applied Biosystems, California, USA). Taqman probes for rat leptin, apelin and 18S rRNA, were also supplied by Applied Biosystems (California, USA). Expression levels were normalized by the mRNA levels of the selected internal control, 18S. All procedures were performed according to protocols described elsewhere ${ }^{19}$.

\section{Statistical analyses}

Student $t$ tests were used for analyzing differences among experimental groups, either for body and biochemical variables of animals, and for measurements performed in isolated adipocytes from rats that were obtained before the primary culture $(n=10)$, or for measurements performed directly on the primary cultures $(n=5)$. On primary cultures, after one-way ANOVA, multiple comparisons using t-student tests were performed at three stages: 1) comparison against control group (without HFD, VC, insulin or HG treatment), 2) comparison against HFD treated group (without VC, insulin or HG treatment), and 3) comparison against HG treated group (without VC or insulin treatment). For correlation analyses, Pearson correlation coefficients were calculated. All results are expressed as means \pm SD. A probability of $\mathrm{p}<0.05$ was set for determining statistically significant differences. All the analyses were performed using the SPSS 15.0 for Windows software (SPSS Inc., Chicago, USA). 


\section{RESULTS}

\section{High fat diet-induced obesity}

The high fat dietary treatment effectively induced overweight in the animals. Thus, HFD treatment induced elevated body and WAT weights, and higher levels of some biochemical blood markers, such as glucose, insulin, and leptin, as compared to rats fed on the chow diet (Table 2). Also, adipocytes isolated from epididymal WAT of HFDfed rats presented different morphological and functional characteristics as compared to controls, such as higher cell diameter and intracellular ROS content, higher apelin and leptin gene expression and lower cell number per suspension volume (ml) (Table 2).

\section{Viability assay}

LDH activity in culture medium after the $72 \mathrm{~h}$ treatment was measured as an indicator of cell viability (Figure 1; one-way ANOVA, $\mathrm{p}<0.001$ ). Cell viability was drastically reduced in adipocytes by HG addition, from both control and HFD treated animals, although more drastically in control adipocytes. This effect was completely reversed by the addition of $\mathrm{VC}$ into the culture media.

\section{Glucose and lipid metabolism}

The glucose uptake, lactate production and glycerol release of adipocytes isolated from epididymal WAT of rats after the $72 \mathrm{~h}$ culture were measured (Figure 2 ). No important effects of HFD treatment on glucose uptake were observed (Figure 2A; one-way ANOVA, $\mathrm{p}<0.01)$. However, insulin treatment, as expected, induced higher glucose uptake, but only in control adipocytes without HG treatment. Although a slight effect of VC was observed, it was only statistically significant in adipocytes from HFD rats. 
Regarding lactate production (Figure 2B; one-way ANOVA, $\mathrm{p}<0.001$ ), insulin induced higher release of this metabolite again only in control adipocytes. This induction was not observed under HG or HFD treatment. On the other hand, VC exerts inhibitory effects in almost all experimental conditions. A slight decrease was observed in adipocytes from HFD fed animals as compared to controls, however without reaching statistical significance $(\mathrm{p}=0.052)$.

Finally, glycerol release was generally reduced in adipocytes from HFD fed rats, and drastically inhibited by VC treatment, especially in primary adipocytes from control animals.

\section{Adipokines mRNA expression}

The mRNA expression of apelin and leptin was also analyzed in the primary cultures (Figure 3). Concerning apelin mRNA expression (Figure 3A; one-way ANOVA, $\mathrm{p}<0.001$ ), no effects of HFD and insulin treatments were observed. VC induced decreased apelin expression, especially in those that were not treated with HG. HG treatment induced a slight reducing effect only in adipocytes from HFD rats $(p=0.094)$. Regarding leptin mRNA expression levels (Figure 3B; one-way ANOVA, $\mathrm{p}<0.001$ ), statistical tendencies towards higher expression induced by insulin $(p=0.068)$ and HG $(p=0.059)$ treatments were only observed in controls. VC treatment induced slight inhibitions in leptin gene expression, only statistically significant in cells under insulin and $\mathrm{HG}$ treatment.

\section{Adipokine secretion}

The secretion of apelin and leptin gene products after $72 \mathrm{~h}$ of culture was also measured

(Figure 4). Insulin treatment induced a statistically significant increase in apelin 
secretion only in control adipocytes (Figure 4A; one-way ANOVA, $<<0.001$ ). On the other hand, HFD treatment induced lower apelin secretion, and HG provoked higher adipokine release, but only in adipocytes from HFD rats. VC treatment overall produced lower apelin secretion, an effect that was particularly significant in control adipocytes. Leptin secretion was significantly induced by insulin only in control adipocytes, regardless of glucose treatment (Figure 4B; one-way ANOVA, $\mathrm{p}<0.001$ ). HG per se induced higher leptin secretion, while VC only inhibited this adipokine in the presence of $\mathrm{HG}$, an effect that was once again only seen in control adipocytes. These effects were blunted in adipocytes from HFD rats.

\section{Association studies}

Correlation analyses among some in vivo and ex vivo measurements were performed (Figure 5). Among variables measured prior to cell culture procedures, intracellular ROS content of adipocytes correlated positively with retroperitoneal WAT weight $(\mathrm{r}=$ 0.673, $\mathrm{p}<0.01)$, mesenteric WAT weight $(\mathrm{r}=0.561, \mathrm{p}<0.05)$, serum leptin concentration $(\mathrm{r}=0.610, \mathrm{p}<0.05)$, and leptin gene expression in adipocytes $(r=0.692, \mathrm{p}<0.01)$. On the other hand, glucose uptake measured after $72 \mathrm{~h}$ of adipocyte culture correlated negatively with several variables measured before culture, such as animal body $(\mathrm{r}=$ 0.387, $\mathrm{p}<0.01)$, epididymal $(\mathrm{r}=-0.316, \mathrm{p}<0.01)$, subcutaneous $(\mathrm{r}=-0.259, \mathrm{p}<0.05)$, retroperitoneal $(\mathrm{r}=-0.398, \mathrm{p}<0.001)$, mesenteric $(\mathrm{r}=-0.354, \mathrm{p}<0.01)$ and total fat depot $(\mathrm{r}=-0.358, \mathrm{p}<0.01)$ weights, blood glucose levels $(\mathrm{r}=-0.230, \mathrm{p}<0.05)$ and adipocyte cell diameter $(r=-0.362, p<0.01)$. Finally, adipocyte apelin gene expression after 72 hours culture correlated positively with rat epididymal fat depot weight $(r=0.327$, $\mathrm{p}<0.01)$ and insulin blood levels $(\mathrm{r}=0.336, \mathrm{p}<0.01)$. 


\section{DISCUSSION}

The scientific literature describes controversial results for a potential role of antioxidants in obesity prevention or treatment ${ }^{26}$. Nevertheless, the health-improving effects of VC treatment on body weight and waist-hip ratio, cardiovascular disease occurrence, and on glucose and lipid biomarkers in humans ${ }^{14,27-29}$ and animals ${ }^{20}$, have been reported. In the present work, some obesity-related variables of adipocytes isolated from obese animals were compared with control adipocytes under VC treatment and subjected to several experimental conditions.

The HFD fed animals presented all features of an obesity-established state. Moreover, the associations observed between intracellular ROS in adipocytes and several obesityrelated variables agree with the previous literature, which reported higher oxidative stress in excessively enlarged adipose tissue. This is an important trigger for the onset of obesity-related metabolic syndrome features ${ }^{9}$. In culture, the onset of insulin resistance is suggested by the fact that lactate release was lower, and that the insulin treatment did not induce higher glucose uptake, lactate production and adipokine release of adipocytes from HFD rats ${ }^{23,30,31}$. In addition, this observation is supported with the negative correlations observed between adipocyte glucose uptake and several obesity-related variables, and with the positive correlations observed between apelin mRNA expression of adipocytes after culture, with WAT weight and blood insulin. On the contrary, lower serum triglycerides levels in HFD fed animals with respect to controls were found. Nevertheless, this result agrees with a previous report performed in the same obesity model $^{32}$. In this study, higher liver triglyceride contents were detected in HFD-fed rats, indicating that this metabolite, at this stage, could be rapidly mobilized to the liver, possibly leading to steatosis. Concerning the higher HDL cholesterol content in HFD 
fed animals, despite the fact that dietary saturated fat intake has been associated with increased plasma cholesterol (particularly LDL), paradoxically, saturated fat intake has also been related with elevated HDLc plasma levels ${ }^{33}$. In this regard, it has been observed that low saturated fat and cholesterol intake reduced apolipoprotein A1 secretion $^{34}$.

Regarding VC treatment, first of all, it is worth mentioning that the dose used had been previously utilized by our research group, and it was described that it is within physiological levels ${ }^{19}$. The facts that insulin-induced adipocytes presented basal glucose uptake levels, and that VC treatment directly inhibits the glucose uptake in adipocytes from HFD animals, agrees with a previous report ${ }^{19}$. Indeed, it is known that the dehydroascorbic acid (the oxidized form of $\mathrm{VC}$ that is transported inside cells) could compete with glucose for GLUT-1 and GLUT-4 transporters ${ }^{35,36}$. The drastic inhibition in lactate production by this vitamin, which was observed in adipocytes from both types, is in accordance with a study describing a lactic acid plasma concentration reduction of dietary feeds by a vitamin $\mathrm{C} / \mathrm{E}$ treatment in diabetic rats ${ }^{37}$, and again, with a previous report that was performed in adipocytes from lean animals ${ }^{19}$. The present work suggests that the effect of this vitamin is independent of the dietary origin of the cells. On the other hand, in muscle cells, a $\mathrm{H}_{2} \mathrm{O}_{2}$ treatment induces glucose uptake, lactate production and the gene expression of proteins involved in glucose metabolism. However, treatment with the antioxidant n-acetylcysteine reduced the ROS content, glucose uptake, lactate production, the expression of GLUT4, and the PFK activity ${ }^{38}$. It was suggested that the reducing properties of n-acetylcysteine on glucose uptake are attributed to its ROS scavenger properties. In this context, VC could also induce glucose uptake inhibition by its well-known ROS scavenging properties ${ }^{35,39}$. 
With regard to lipolytic activity, the lower glycerol release of adipocytes from HFD-fed rats could be due to a fat hydrolysis resistance ${ }^{40,41}$. On the other hand, despite the fact that the results did not replicate the well-known antilipolytic effects of insulin ${ }^{42}$, these are in agreement with other reports ${ }^{19,43}$, indicating a lack of lipolysis modulation by insulin in this experimental model. Also, it is worth mentioning that the antilipolytic effects of insulin are blunted in visceral compared to subcutaneous adipocytes ${ }^{44}$. Finally, the lower glycerol release induced by VC indicates an inhibited fat utilization on both insulin-treated and non-treated adipocytes from chow or HFD-fed rats. It has been described that rats fed on HFD with VC supplementation presented a decreased isoproterenol-induced lipolysis as compared with the rats that were fed with high fat diet alone ${ }^{15}$, and that VC inhibited glycerol release in primary cultured adipocytes from lean animals ${ }^{19}$. Furthermore, the lower lipolysis is in agreement with a possible insulinsensitizing property of $\mathrm{VC}^{13,15,29}$.

Regarding apelin and leptin expression and secretion, at first glance, no positive correlations between the expression pattern and the secretion levels of both adipokines were observed. This outcome could be explained by possible mRNA expression fluctuations during culture and by probable post-transcriptional events ${ }^{45,46}$. As mentioned before, the lower apelin and leptin secretion response to insulin in adipocytes from HFD-fed rats could be attributed to the insulin-resistant state of these adipocytes ${ }^{23}$. It is important to notice, that this effect was overcome by the HG treatment especially in the apelin secretion, which agrees with a previous report ${ }^{47}$. Leptin production appears to be more blunted by the HFD treatment. With respect to the effects of VC, the results are consistent with a previously described inhibition of apelin expression and leptin expression/secretion in WAT from rats that were fed on a high fat diet supplemented with $\mathrm{VC}^{13}$. The present work confirms a direct effect of $\mathrm{VC}$ treatment in adipocytes that 
were isolated from rat visceral fat. These data show that the $\mathrm{VC}$ treatment leads to important secretion alterations independent of the dietary origin of the adipocytes. The VC inhibitory effects on leptin secretion are in accordance with a study describing direct inhibitory effects in adipocytes isolated from lean rats ${ }^{19}$. Similar results were observed with other antioxidant substances, such as resveratrol and lipoic acid ${ }^{48,49}$. Regarding the possible mechanisms that could have lead to the observed results, it has been reported that a VC treatment reduced the hypoxia-inducible factor $1 \alpha(\mathrm{HIF}-1 \alpha)$ expression in rat prostate $^{50}$, factor which is known to induce apelin gene expression ${ }^{51}$. Also, it has been reported that insulin and hypoxia, through HIF-1 $\alpha$, act as agonists of leptin transcription in BeWo cells ${ }^{52}$. Moreover, both genes present insulin-response elements in their promoters ${ }^{53,54}$, and both transcripts could be affected by ROS content $t^{55}$.

A toxic effect of HG incubation was observed in the current experiments, as was reported previously in the same experimental model ${ }^{56}$. Although it has been previously described that an in vivo VC treatment reduced adipocyte cell number ${ }^{17}$, no negative effects on ex vivo cell viability of this vitamin were detected. In fact, we observed a protective effect for this molecule against HG toxicity. This finding could be due to competition with glucose for GLUT-1, and -4 transporters ${ }^{35,36}$, reducing the effects of glucose on the hexosamine flux pathway ${ }^{57}$. Consequently, this situation could avoid cell death in control cells and possibly improve insulin sensitivity, since glucose toxicity contributes to insulin-resistance ${ }^{58}$. In this context, it has previously been found that antioxidant treatments reduce glucose toxicity in pancreatic $\beta$ cells (HIT-T15) as well as in Zucker diabetic obese rats ${ }^{59}$, and that cells under HG treatment present augmented ROS intracellular content and suffer mitochondrial dysfunction ${ }^{60}$. Finally, this could 
explain the relatively lower effect of VC on adipocytes from HFD animals, since these cells presented higher ROS content prior to culture.

In summary, adipocytes isolated from the epididymal fat of rats, which were fed on either a chow or high fat diet, presented different responses to glucose and insulin incubations. Animals in each group displayed differences in their levels of glucose and lipid metabolism markers, as well as in their expression/secretion pattern of apelin and leptin in primary culture, due to the establishment of an insulin-resistant state in animals fed on HFD. Furthermore, vitamin C incubation in this culture leads to dramatic modifications of these variables, although these effects appear to be independent of the dietary origin of the adipocytes. Indeed, primary adipocytes from animals fed on HFD diet show impairment in metabolic functions concerning glucose and lipid metabolism as compared to chow fed animals, while the response to $\mathrm{VC}$ appears to be inhibitory in both dietary groups. 


\section{AUTHOR CONTRIBUTIONS}

DFGD, JC, FIM, MJMA and JAM participated in the experimental design. DFGD and AVA performed the experimental procedures and the data collection and analysis. All authors participated in the data interpretation. DFGD and JC wrote the manuscript. FIM, MJMA and JAM reviewed the manuscript.

\section{ACKNOWLEDGEMENTS}

This work was supported by the Ministry for Education and Science (MEC, Spain, grant AGL2006-04716/ALI), the Education Department of the Navarra Government (Spain), Carlos III Health Institute (CIBER project, Spain, grant CB06/03/1017; and RETICS project, Spain, PREDIMED2), the "Línea Especial” (LE/97, University of Navarra, Spain), and the "Asociación de Amigos de la Universidad de Navarra" and IBERCAJA (Spain, DFGD doctoral grants). Also, the authors wish to thank the Department of Biochemistry of the University of Navarra for kindly lending us the POLARstar spectrofluorometer plate reader for ROS concentration measurements. Also, the authors are grateful for the expert technical assistance of Ana Lorente and Veronica Ciaurriz. 


\section{REFERENCES}

1. Bray GA. Medical consequences of obesity. J Clin Endocrinol Metab 2004;89:2583-9.

2. Ferrante AW. Obesity-induced inflammation: a metabolic dialogue in the language of inflammation. J Intern Med 2007;262:408-14.

3. Fantuzzi G. Adipose tissue, adipokines, and inflammation. J Allergy Clin Immunol 2005;115:911-9.

4. Zhang Y, Proenca R, Maffei M, Barone M, Leopold L, Friedman JM. Positional cloning of the mouse obese gene and its human homologue. Nature 1994;372:425-32.

5. Boucher J, Masri B, Daviaud D, Gesta S, Guigne C, Mazzucotelli A, CastanLaurell I, Tack I, Knibiehler B, Carpene C, Audigier Y, Saulnier-Blache JS, Valet P. Apelin, a newly identified adipokine up-regulated by insulin and obesity. Endocrinology 2005;146:1764-71.

6. Daviaud D, Boucher J, Gesta S, Dray C, Guigne C, Quilliot D, Ayav A, Ziegler O, Carpene C, Saulnier-Blache JS, Valet P, Castan-Laurell I. TNFalpha up-regulates apelin expression in human and mouse adipose tissue. FASEB J 2006;20:1528-30.

7. Martinez JA. Mitochondrial oxidative stress and inflammation: an slalom to obesity and insulin resistance. J Physiol Biochem 2006;62:303-6.

8. Vincent HK, Taylor AG. Biomarkers and potential mechanisms of obesityinduced oxidant stress in humans. Int J Obes 2006;30:400-18.

9. Furukawa S, Fujita T, Shimabukuro M, Iwaki M, Yamada Y, Nakajima Y, Nakayama O, Makishima M, Matsuda M, Shimomura I. Increased oxidative stress in obesity and its impact on metabolic syndrome. J Clin Invest 2004;114:1752-61. 
10. Moreno-Aliaga MJ, Campión J, Milagro FI, Berjón A, Martínez JA. Adiposity and proinflammatory state: The chicken or the egg. Adipocytes 2005;1:1-9.

11. Bsoul SA, Terezhalmy GT. Vitamin C in health and disease. J Contemp Dent Pract 2004;5:1-13.

12. Campion J, Milagro FI, Fernandez D, Martinez JA. Diferential gene expression and adiposity reduction induced by ascorbic acid supplementation in a cafeteria model of obesity. J Physiol Biochem 2006;62:71-80.

13. Garcia-Diaz D, Campion J, Milagro FI, Martinez JA. Adiposity dependent apelin gene expression: relationships with oxidative and inflammation markers. Mol Cell Biochem 2007;305:87-94.

14. Johnston CS, Beezhold BL, Mostow B, Swan PD. Plasma vitamin C is inversely related to body mass index and waist circumference but not to plasma adiponectin in nonsmoking adults. $J$ Nutr 2007;137:1757-62.

15. Garcia-Diaz DF, Campion J, Milagro FI, Paternain L, Solomon A, Martinez JA. Ascorbic acid oral treatment modifies lipolytic response and behavioural activity but not glucocorticoid metabolism in cafeteria diet-fed rats. Acta Physiol (Oxf) 2009;195:44957.

16. Hasegawa N, Niimi N, Odani F. Vitamin C is one of the lipolytic substances in green tea. Phytother Res 2002;16 Suppl 1:S91-2.

17. Senen D, Adanali G, Ayhan M, Gorgu M, Erdogan B. Contribution of vitamin C administration for increasing lipolysis. Aesthetic Plast Surg 2002;26:123-5.

18. Doulas NL, Constantopoulos A, Litsios B. Effect of ascorbic acid on guinea pig adrenal adenylate cyclase activity and plasma cortisol. J Nutr 1987;117:1108-14. 
19. Garcia-Diaz DF, Campion J, Milagro FI, Boque N, Moreno-Aliaga MJ, Martinez JA. Vitamin C inhibits leptin secretion and some glucose/lipid metabolic pathways in primary rat adipocytes. $J$ Mol Endocrinol 2010;45:33-43.

20. Abdel-Wahab YH, O'Harte FP, Mooney MH, Barnett CR, Flatt PR. Vitamin C supplementation decreases insulin glycation and improves glucose homeostasis in obese hyperglycemic (ob/ob) mice. Metabolism 2002;51:514-7.

21. Carcamo JM, Pedraza A, Borquez-Ojeda O, Golde DW. Vitamin C suppresses TNF alpha-induced NF kappa B activation by inhibiting I kappa B alpha phosphorylation. Biochemistry 2002;41:12995-3002.

22. Garcia-Diaz DF, Campion J, Quintero P, Milagro FI, Moreno-Aliaga MJ, Martinez JA. Vitamin $\mathrm{C}$ modulates the interaction between adipocytes and macrophages. Mol Nutr Food Res 2011;55 Suppl 2:S257-63.

23. Boque N, Campion J, Milagro FI, Moreno-Aliaga MJ, Martinez JA. Some cyclin-dependent kinase inhibitors-related genes are regulated by vitamin $\mathrm{C}$ in a model of diet-induced obesity. Biol Pharm Bull 2009;32:1462-8.

24. Racher AJ, Looby D, Griffiths JB. Use of lactate dehydrogenase release to assess changes in culture viability. Cytotechnology 1990;3:301-7.

25. Campion J, Martinez JA. Ketoconazole, an antifungal agent, protects against adiposity induced by a cafeteria diet. Horm Metab Res 2004;36:485-91.

26. Valdecantos MP, Perez-Matute P, Martinez JA. Obesity and oxidative stress: role of antioxidant supplementation. Rev Invest Clin 2009;61:127-39.

27. Canoy D, Wareham N, Welch A, Bingham S, Luben R, Day N, Khaw KT. Plasma ascorbic acid concentrations and fat distribution in 19,068 British men and women in the European Prospective Investigation into Cancer and Nutrition Norfolk cohort study. Am J Clin Nutr 2005;82:1203-9. 
28. Kurl S, Tuomainen TP, Laukkanen JA, Nyyssonen K, Lakka T, Sivenius J, Salonen JT. Plasma vitamin C modifies the association between hypertension and risk of stroke. Stroke 2002;33:1568-73.

29. Paolisso G, Balbi V, Volpe C, Varricchio G, Gambardella A, Saccomanno F, Ammendola S, Varricchio M, D'Onofrio F. Metabolic benefits deriving from chronic vitamin $\mathrm{C}$ supplementation in aged non-insulin dependent diabetics. J Am Coll Nutr 1995;14:387-92.

30. Lovejoy J, Newby FD, Gebhart SS, DiGirolamo M. Insulin resistance in obesity is associated with elevated basal lactate levels and diminished lactate appearance following intravenous glucose and insulin. Metabolism 1992;41:22-7.

31. Graham TE, Kahn BB. Tissue-specific alterations of glucose transport and molecular mechanisms of intertissue communication in obesity and type 2 diabetes. Horm Metab Res 2007;39:717-21.

32. Lomba A, Martinez JA, Garcia-Diaz DF, Paternain L, Marti A, Campion J, Milagro FI. Weight gain induced by an isocaloric pair-fed high fat diet: a nutriepigenetic study on FASN and NDUFB6 gene promoters. Mol Genet Metab 2010;101:273-8.

33. Mensink RP, Zock PL, Kester AD, Katan MB. Effects of dietary fatty acids and carbohydrates on the ratio of serum total to HDL cholesterol and on serum lipids and apolipoproteins: a meta-analysis of 60 controlled trials. Am J Clin Nutr 2003;77:114655.

34. Velez-Carrasco W, Lichtenstein AH, Welty FK, Li Z, Lamon-Fava S, Dolnikowski GG, Schaefer EJ. Dietary restriction of saturated fat and cholesterol decreases HDL ApoA-I secretion. Arterioscler Thromb Vasc Biol 1999;19:918-24. 
35. Arrigoni O, De Tullio MC. Ascorbic acid: much more than just an antioxidant. Biochim Biophys Acta 2002;1569:1-9.

36. Vera JC, Rivas CI, Fischbarg J, Golde DW. Mammalian facilitative hexose transporters mediate the transport of dehydroascorbic acid. Nature 1993;364:79-82. 37. Ruperez FJ, Garcia-Martinez D, Baena B, Maeso N, Cifuentes A, Barbas C, Herrera E. Evolution of oxidative stress parameters and response to oral vitamins $\mathrm{E}$ and C in streptozotocin-induced diabetic rats. J Pharm Pharmacol 2008;60:871-8. 38. Pinheiro CH, Silveira LR, Nachbar RT, Vitzel KF, Curi R. Regulation of glycolysis and expression of glucose metabolism-related genes by reactive oxygen species in contracting skeletal muscle cells. Free Radic Biol Med 2010;48:953-60. 39. Perticone F, Ceravolo R, Candigliota M, Ventura G, Iacopino S, Sinopoli F, Mattioli PL. Obesity and body fat distribution induce endothelial dysfunction by oxidative stress: protective effect of vitamin C. Diabetes 2001;50:159-65.

40. Arner P. Catecholamine-induced lipolysis in obesity. Int J Obes Relat Metab Disord 1999;23 Suppl 1:10-3.

41. Jocken JW, Blaak EE. Catecholamine-induced lipolysis in adipose tissue and skeletal muscle in obesity. Physiol Behav 2008;94:219-30.

42. Elks ML, Manganiello VC. Antilipolytic action of insulin: role of cAMP phosphodiesterase activation. Endocrinology 1985;116:2119-21.

43. Perez-Matute P, Marti A, Martinez JA, Fernandez-Otero MP, Stanhope KL, Havel PJ, Moreno-Aliaga MJ. Conjugated linoleic acid inhibits glucose metabolism, leptin and adiponectin secretion in primary cultured rat adipocytes. Mol Cell Endocrinol 2007;268:50-8.

44. Wu X, Hoffstedt J, Deeb W, Singh R, Sedkova N, Zilbering A, Zhu L, Park PK, Arner P, Goldstein BJ. Depot-specific variation in protein-tyrosine phosphatase 
activities in human omental and subcutaneous adipose tissue: a potential contribution to differential insulin sensitivity. J Clin Endocrinol Metab 2001;86:5973-80.

45. Bradley RL, Cheatham B. Regulation of ob gene expression and leptin secretion by insulin and dexamethasone in rat adipocytes. Diabetes 1999;48:272-8.

46. Lorente-Cebrian S, Bustos M, Marti A, Martinez JA, Moreno-Aliaga MJ. Eicosapentaenoic acid up-regulates apelin secretion and gene expression in 3T3-L1 adipocytes. Mol Nutr Food Res 2010;54 Suppl 1:S104-11.

47. Dray C, Knauf C, Daviaud D, Waget A, Boucher J, Buleon M, Cani PD, Attane C, Guigne C, Carpene C, Burcelin R, Castan-Laurell I, Valet P. Apelin stimulates glucose utilization in normal and obese insulin-resistant mice. Cell Metab 2008;8:43745.

48. Szkudelska K, Nogowski L, Szkudelski T. The inhibitory effect of resveratrol on leptin secretion from rat adipocytes. Eur J Clin Investig 2009;39:899-905.

49. Prieto-Hontoria PL, Perez-Matute P, Fernandez-Galilea M, Martinez JA, Moreno-Aliaga MJ. Lipoic acid inhibits leptin secretion and Sp1 activity in adipocytes. Mol Nutr Food Res 2011;55:1059-69.

50. Li SH, Ryu JH, Park SE, Cho YS, Park JW, Lee WJ, Chun YS. Vitamin C supplementation prevents testosterone-induced hyperplasia of rat prostate by downregulating HIF-1alpha. J Nutr Biochem 2010;21:801-8.

51. Glassford AJ, Yue P, Sheikh AY, Chun HJ, Zarafshar S, Chan DA, Reaven GM, Quertermous T, Tsao PS. HIF-1 regulates hypoxia- and insulin-induced expression of apelin in adipocytes. Am J Physiol Endocrinol Metab 2007;293:E1590-6.

52. Meissner U, Ostreicher I, Allabauer I, Rascher W, Dotsch J. Synergistic effects of hypoxia and insulin are regulated by different transcriptional elements of the human leptin promoter. Biochem Biophys Res Commun 2003;303:707-12. 
53. Moreno-Aliaga MJ, Swarbrick MM, Lorente-Cebrian S, Stanhope KL, Havel PJ, Martinez JA. Sp1-mediated transcription is involved in the induction of leptin by insulin-stimulated glucose metabolism. J Mol Endocrinol 2007;38:537-46.

54. Wang G, Qi X, Wei W, Englander EW, Greeley GH, Jr. Characterization of the 5'-regulatory regions of the rat and human apelin genes and regulation of breast apelin by USF. FASEB J 2006;20:2639-41.

55. Pessler D, Rudich A, Bashan N. Oxidative stress impairs nuclear proteins binding to the insulin responsive element in the GLUT4 promoter. Diabetologia 2001;44:2156-64.

56. Garcia-Diaz DF, Arellano AV, Milagro FI, Moreno-Aliaga MJ, Portillo MP, Martinez JA, Campion J. Glucose and insulin modify thrombospondin 1 expression and secretion in primary adipocytes from diet-induced obese rats. J Physiol Biochem 2011;67:453-61.

57. Teo CF, Wollaston-Hayden EE, Wells L. Hexosamine flux, the O-GlcNAc modification, and the development of insulin resistance in adipocytes. Mol Cell Endocrinol 2010;318:44-53.

58. Rossetti L, Giaccari A, DeFronzo RA. Glucose toxicity. Diabetes Care 1990;13:610-30.

59. Tanaka Y, Gleason CE, Tran PO, Harmon JS, Robertson RP. Prevention of glucose toxicity in HIT-T15 cells and Zucker diabetic fatty rats by antioxidants. Proc Natl Acad Sci U S A 1999;96:10857-62.

60. Gao CL, Zhu C, Zhao YP, Chen XH, Ji CB, Zhang CM, Zhu JG, Xia ZK, Tong ML, Guo XR. Mitochondrial dysfunction is induced by high levels of glucose and free fatty acids in 3T3-L1 adipocytes. Mol Cell Endocrinol 2010;320:25-33. 


\section{FIGURE LEGENDS}

Figure 1. Viability assay of the primary culture. The effects of $200 \mu \mathrm{M}$ vitamin C, 1.6 $\mathrm{nM}$ insulin, and high $(25 \mathrm{mM})$ or low $(5.6 \mathrm{mM})$ glucose concentrations on $72 \mathrm{~h}$ lactate dehydrogenase activity in culture medium of isolated adipocytes from chow (white bars) of high fat diet (black bars) fed rats are shown. Data $(n=5)$ are expressed as median $\pm \mathrm{SD} .{ }^{* * *} \mathrm{p}<0.001$ indicates differences against control group; ${ }^{\dagger} \mathrm{p}<0.05$ against HFD treated-only group; ${ }^{\S} \mathrm{p}<0.05,{ }^{\S \S} \mathrm{p}<0.01$ and ${ }^{\S \S} \mathrm{p}<0.001$ against each (control and HFD) HG treated group. HF, high fat; VC, vitamin C; INS, insulin; HG, high glucose.

Figure 2. Glucose and lipid metabolism measurements in the primary culture. The effects of $200 \mu \mathrm{M}$ vitamin C, $1.6 \mathrm{nM}$ insulin, and high (25 mM) or low (5.6 mM) glucose concentrations on $72 \mathrm{~h}$ (A) glucose uptake, (B) lactate production, and (C) glycerol release of isolated adipocytes from chow (white bars) of high fat diet (black bars) fed rats are shown. Data $(n=5)$ are expressed as median \pm SD. ${ }^{*} \mathrm{p}<0.05$ and $* * p<0.01$ indicate differences against control group; ${ }^{\dagger} \mathrm{p}<0.05$ against HFD treated-only group; ${ }^{\S} \mathrm{p}<0.05$ against each (control and HFD) HG treated group. HF, high fat; VC, vitamin $\mathrm{C}$; INS, insulin; HG, high glucose; $\mathrm{t}^{*}$, statistical tendency.

Figure 3. Adipokines gene expression measurements in the primary culture. The effects of $200 \mu \mathrm{M}$ vitamin C, $1.6 \mathrm{nM}$ insulin, and high (25 mM) or low (5.6 mM) glucose concentrations on $72 \mathrm{~h} \mathrm{(A)} \mathrm{apelin,} \mathrm{and} \mathrm{(B)} \mathrm{leptin} \mathrm{mRNA} \mathrm{expression} \mathrm{of} \mathrm{isolated}$ adipocytes from chow (white bars) of high fat diet (black bars) fed rats are shown. Data $(\mathrm{n}=5)$ are expressed as median \pm SD. ${ }^{*} \mathrm{p}<0.05$ indicates differences against control

group; ${ }^{\dagger \dagger} \mathrm{p}<0.01$ against HFD treated-only group; ${ }^{\S} \mathrm{p}<0.05$ against each (control and 
HFD) HG treated group. HF, high fat; VC, vitamin C; INS, insulin; HG, high glucose; $t^{*}$, statistical tendency.

Figure 4. Adipokines secretion measurements in the primary culture. The effects of 200 $\mu \mathrm{M}$ vitamin $\mathrm{C}, 1.6 \mathrm{nM}$ insulin, and high $(25 \mathrm{mM})$ or low $(5.6 \mathrm{mM})$ glucose concentrations on $72 \mathrm{~h}(\mathrm{~A})$ apelin, and (B) leptin secretion to culture medium of isolated adipocytes from chow (white bars) of high fat diet (black bars) fed rats are shown. Data $(\mathrm{n}=5)$ are expressed as median \pm SD. ${ }^{*} \mathrm{p}<0.05, * * \mathrm{p}<0.01$ and $* * * \mathrm{p}<0.001$ indicate differences against control group; ${ }^{\dagger \dagger} p<0.01$ against HFD treated-only group; ${ }^{\S} \mathrm{p}<0.05$ and ${ }^{\S} \mathrm{p}<0.01$ against each (control and HFD) HG treated group. HF, high fat; VC, vitamin C; INS, insulin; HG, high glucose; $t^{*}$, statistical tendency.

Figure 5. Association analyses between intracellular ROS content prior to cell culture and (A) retroperitoneal WAT weight, (B) mesenteric WAT weight, (C) serum leptin and (D) adipocyte leptin gene expression prior to cell culture, between glucose uptake after cell culture and (E) final body weight, $(F)$ total fat weight, $(G)$ serum glucose and $(H)$ adipose cell diameter prior to cell culture, and between apelin gene expression after cell culture and (I) epididymal WAT weight and (J) serum insulin. Triangles represent control fed animals and closed circles represent HFD fed animals. AU, arbitrary units, FI, fluorescence intensity; WAT, white adipose tissue; r, Pearson's correlation coefficient. 